\title{
Syphilis testing among spouses of patients with syphilis in Japan: an epidemiological study using an administrative claims database
}

\author{
$\operatorname{AUTHOR}(\mathrm{S}):$
}

Matsubayashi, Keisuke; Kawakami, Koji

\section{CITATION:}

Matsubayashi, Keisuke ...[et al]. Syphilis testing among spouses of patients with syphilis in Japan: an epidemiological study using an administrative claims database. International Journal of STD \& AIDS 2020, 31(3): 214-220

\section{ISSUE DATE:}

2020-03-01

URL:

http://hdl.handle.net/2433/250800

\section{RIGHT:}

This is an accepted manuscript of the following article: International Journal of STD \& AIDS, 31(3), pp. 214-220. Copyright (c) The Author(s) 2020. DOI:

10.1177/0956462419892779.; この論文は出版社版でありません。引用の際には出版社版 をご確認ご利用ください。; This is not the published version. Please cite only the published version. 
First edition

1 Title page

2

3 Syphilis testing among spouses of patients with syphilis in Japan: an epidemiological

4 study using an administrative claims database

5

6 Keisuke Matsubayashi, MD, MSc${ }^{1}$; Koji Kawakami, MD, $\mathrm{PhD}^{1}$,

$7{ }^{1}$ Department of Pharmacoepidemiology, Graduate School of Medicine and Public Health,

8 Kyoto University, Yoshida-konoecho, Sakyo-ku, Kyoto 606-8501, Japan;

9

10 Address for correspondence 
First edition

11 Koji Kawakami, MD, PhD

12 Department of Pharmacoepidemiology, Graduate School of Medicine and Public Health,

13 Kyoto University, Yoshida- Konoe-cho, Sakyo-ku, Kyoto 606-8501, Japan

14 Tel: +81- 75-753-9469, Fax: +81-75-753-4469

15 Email: kawakami.koji.4e@kyoto-u.ac.jp

16

17 Word count: Abstract: 199 words; Text: 2,998 words; 31 references; 3 tables; 1 figure

18

19 
Second edition

1 Introduction

2 Incidence rates of syphilis, a sexually transmitted infection (STI) caused by Treponema

3 pallidum, is increasing in high-income countries. ${ }^{1,2}$ In the United States and Western Europe,

4 there has been a sharp increase in cases of primary and secondary syphilis primarily among

5 men who have sex with men. ${ }^{1,2}$ In Japan, the incidence of syphilis increased from 883 in 2012

6 to 4564 in 2016, and the majority of syphilis cases were among heterosexual men and

7 women. ${ }^{3}$ In addition, among young women, the number of primary and secondary syphilis

8 cases reported in Japan has increased rapidly since 2014, with the proportion of women

9 among those aged $20-29$ years increasing from $31.1 \%$ in 2014 to $54.5 \%$ in $2018.4,5$ Syphilis

10 in women of reproductive age, especially those in their 20 s and 30 s, can lead to mother-to- 


\section{Second edition}

11 child transmission during pregnancy and adverse pregnancy outcomes, including stillbirth,

12 neonatal death, low birth-weight, and prematurity. Therefore, public health strategies need to

13 be strengthened to prevent the spread of syphilis, especially through heterosexual contact

14 among women of reproductive age.

15 Partner notification $(\mathrm{PN})$ is one of the most critical public health interventions for

16 prevention of the spread of STIs. PN is a means for informing the sex partners of patients

17 diagnosed with an STI that they have been exposed to an STI and for providing testing and

18 treatment. There are five main PN strategies: (1) patient referral, (2) assisted partner

19 notification, (3) expedited partner therapy, (4) provider referral, and (5) contact referral. ${ }^{6}$ To

20 eradicate infection and prevent re-infection, many high-income countries adopt multiple 
Second edition

21 types of PN in their programs for syphilis control. ${ }^{7-10}$ However, of these strategies, only

22 simple patient referral, which entails a physician advising patients that their partner should

23 be tested, is available in Japan. This is primarily because personal information that can

24 identify patients with syphilis is not included in the items reported by physicians to local

25 public health centres under the Infectious Diseases Act. As a result, staff working in local

26 public health centres cannot interview patients or notify their partners. With the substantial

27 increase in the incidence of syphilis, PN has become a more critical means of controlling

28 syphilis; therefore, public health policymakers need to understand the effectiveness of simple

29 patient referral in Japan. However, it remains unclear, whether contacts of patients with

30 syphilis are notified that they are at risk of syphilis transmission, and whether contacts receive 
Second edition

31 treatment for syphilis.

32 Infection control policies should be reorganised in response to the growing syphilis

33 epidemic. ${ }^{11}$ If investigations reveal that few contacts with syphilis have been screened

34 through simple patient referral, Japanese policymakers may try to modernise current

35 infection control strategies to provide a more practical partner service. In the present study,

36 we aimed to determine the proportion of patients whose spouses, particularly women of

37 reproductive age, underwent syphilis testing after their partners were diagnosed with syphilis,

38 using an administrative claims database. 
Second edition

41 Study design and data source

42 We conducted a retrospective descriptive study that used a large administrative claims

43 database maintained by the Japan Medical Data Center (JMDC, Tokyo, Japan). The analysis

44 covered claims between January 2010 and December 2017. The JMDC collects data

45 regarding inpatient, outpatient, and pharmacy insurance claims of members of various health

46 insurance associations that cover employees in large companies, and the spouses and

47 dependents of these members. ${ }^{12,13}$ The database includes clinical and procedural information

48 with an encrypted personal identifier, sex, year and month of birth, medical diagnosis codes

49 according to the International Classification of Diseases 10th Revision (ICD-10), the drug 


\section{Second edition}

50 codes according to the World Health Organization (WHO) Anatomical Therapeutic

51 Chemical system, and the medical procedure codes. The database also includes anonymised

52 family identification codes and insurance coverage periods, which enabled us to identify

53 husband-wife relationships according to the time period. Due to the JMDC's privacy policy,

54 medical diagnosis codes, drug codes, and medical procedure codes linked with human

55 immunodeficiency virus infections were not stored in the database and were not provided to

56 the investigators.

58 approved by the Institutional Review Board of the University of Kyoto (No. R1563). Due to

59 the anonymous nature of the data, the requirement for informed consent was waived in 
Second edition

60 accordance with the Japanese ethical guidelines.

61

\section{Syphilis testing in Japan}

63 Syphilis testing in Japan is divided into those tests that are covered by health insurance and

64 those that are not. The tests covered by health insurance are performed at medical institutions

65 on patients with symptoms or suspected infection. In addition, tests for infection, including

66 syphilis testing, are performed before invasive treatment, such as surgery. Conversely, the

67 tests not covered by health insurance include free tests conducted at local public health

68 centres and universal prenatal syphilis screening at an early gestational age. Routine annual

69 general health check-ups do not include syphilis testing. 
Second edition

70

71 Selection of index patients with syphilis and their spouses

72 We identified index patients who were newly treated for syphilis between January 2010 and

73 December 2017. The dates of syphilis treatment were determined based on the month of the

74 first prescription of antibiotics for syphilis. New treatment of syphilis was defined as (1)

75 having a definitive diagnosis of syphilis (ICD-10 code: A51, A52, or A53); (2) prescription

76 of antibiotics for syphilis; and (3) performance of syphilis testing as both nontreponemal and

77 treponemal tests in the month of antibiotic prescription, or in the month preceding antibiotic

78 prescription. Antibiotics for syphilis were defined according to the guidelines of the Japanese

79 Society for Sexually Transmitted Infections (amoxicillin, ampicillin, minocycline, 


\section{Second edition}

80 doxycycline, azithromycin, acetylspiramycin, ceftriaxone, and benzylpenicillin). ${ }^{14,15}$ The

81 guidelines suggest using amoxicillin or ampicillin $500 \mathrm{mg}$ three times daily orally.

82 Benzathine penicillin $\mathrm{G}$, which is recommended by the WHO guidelines for the treatment of

83 Treponema pallidum infection, ${ }^{16}$ was not included in the list because it has not been available

84 in Japan.

85 We excluded patients aged $<20$ years because this database only identified married

86 pairs, and adolescents are unlikely to be married. To increase the accuracy of the definition

87 of newly treated syphilis by excluding patients treated previously, we required continuous

88 enrolment in the health insurance plan for at least 6 months before the syphilis treatment

89 started. We also excluded patients who underwent surgery, blood transfusion, gastrointestinal 
Second edition

90 endoscopy, or coronary angiography during the index month because syphilis testing may be

91 performed as a screening test for these procedures.

92 We identified the spouses of index patients using anonymised family identification

93 codes. If both the husband and wife were treated for syphilis, we chose the spouse who was

94 treated first as the index patient. To determine whether the spouse was tested after treatment

95 of the index patient, we included only spouses who were enrolled in the health insurance plan

96 for at least three months after the index patient started treatment.

97

$98 \quad$ Outcomes

99 The outcome of interest was spousal syphilis testing within three months of the index patient 
Second edition

100 starting syphilis treatment. Spousal syphilis testing was defined as either nontreponemal or

101 treponemal testing for syphilis according to the medical procedure codes. Because the dataset

102 used in this study did not include information on whether the index patient notified his or her

103 spouse, spousal notification was not an outcome of this study.

104

105 Other variables

106 We extracted information on index patient demographic characteristics, including age (20-

10744 and 45-74 years), sex, and clinical characteristics of previous STI diagnoses according to

108 the ICD-10 codes (including gonorrhoea (A54), chlamydia (A55-A56), genital herpes (A60)),

109 and psychiatric disorders (schizophrenia, schizotypal and delusional disorders: F20-F29 and 
Second edition

110 mood disorders: F30-F39). Clinical data within 3 months preceding the index month were

111 also assessed. We also recorded the provider type (clinic and hospital) and the year of syphilis

112 treatment (2010-2011, 2012-2013, 2014-2015, and 2016-2017). In Japan, clinics are

113 defined as medical institutions with 19 beds or fewer, and hospitals as those with 20 beds or

114 more. Spousal demographic characteristics included age (20-44 and 45-74 years) and sex.

\section{5}

\section{Statistical analyses}

117 Firstly, descriptive statistics were calculated for the basic demographic and clinical variables

118 of the index patient. Secondly, male-female and female-male contacts were analysed

119 separately. We calculated the proportion of patients with newly treated syphilis during the 
Second edition

120 study period whose spouses had undergone syphilis testing within 3 months of treatment

121 initiation. Associations between the characteristics of index patients and their spouses and

122 performance of spousal syphilis testing were evaluated using Fisher's exact test. The trend

123 in the proportion of spousal syphilis testing during the study period was assessed using the

124 Cochran-Armitage trend test. Thirdly, to evaluate the prevention of congenital syphilis, we

125 determined the proportion of men married to women aged 20-44 years who had undergone

126 a spousal syphilis test. This age range was chosen because approximately $99 \%$ of mothers in

127 Japan gave birth when they were between the ages of 20 and 44 years. ${ }^{17}$ Probability values

128 for statistical tests were two-tailed, and p-values $<0.05$ were considered to be statistically

129 significant. All statistical analyses were performed using SAS version 9.4 for Windows (SAS 
Second edition

130 Institute, Cary, NC, USA). 
Second edition

\section{Results}

132 During the study period, 217 pairs of patients with syphilis and their spouses met the

133 inclusion criteria (Figure 1). The characteristics of the index patients with syphilis are shown

134 in Table 1. Between 2010 and 2013, 10 or fewer index patients were diagnosed with syphilis

135 per year; however, during the period 2014-2017, the number of index patients increased each

136 year from 23 in 2014 to 75 in 2017.

138 the index patient's new syphilis treatment (Table 2). The proportion of spousal syphilis

139 testing was $12.6 \%$ among the wives of male index patients, and $17.1 \%$ among the husbands

140 of female index patients, respectively. Among male index patients, the proportion whose 
Second edition

141 wives were tested was higher among those aged 20-44 years than among those aged 45-74

142 years, whereas among female index patients, the proportion whose husbands were tested did

143 not differ by age group. The proportion of spouses tested did not differ significantly

144 according to STI history or psychiatric disease history. The spouses of all 12 index patients

145 who were treated at a hospital were tested for syphilis.

146 Although not significant, among male index patients, the wives of those aged 20-44

147 years were more likely to undergo syphilis testing than those aged 45-74 years. Similarly,

148 the wives aged 20-44 years were more likely to undergo syphilis testing than those aged 45-

14974 years. The proportion of spousal syphilis testing declined from $37.5 \%$ in $2010-2011$ to

$1509.5 \%$ in $2016-2017(P$ for trend $=0.01)$. 
Second edition

151 Table 3 shows the proportion of wives aged 20-44 years who underwent spousal

152 syphilis testing. The proportion of spousal syphilis testing was higher among index patients

153 aged 20-44 years than among those aged 45-74 years. The proportion of spousal syphilis

154 testing decreased significantly between 2010-2011 and 2016-2017 $(P$ for trend $=0.03)$. 
Second edition

\section{Discussion}

156 To the best of our knowledge, the present study is the first in Japan to describe partner syphilis

157 testing using an administrative claims database. Less than one in seven spouses of index

158 patients with syphilis underwent syphilis testing within three months after their spouse was

159 treated. In the subgroup of wives aged 20-44 years, who constitute women of reproductive

160 age, approximately one-fifth of underwent syphilis testing within three months, and the

161 proportion tested decreased significantly between 2011-2012 and 2016-2017. Another

162 unique feature of our study is the use of existing health insurance claims data without the

163 collection of primary data. We determined whether partners were tested using claims data

164 which identified married couples. 
Second edition

166 partners of patients, who were diagnosed with an STI such as chlamydia or gonorrhoea, were

167 examined or treated for STIs. ${ }^{18}$ Recent studies conducted in the United States reported that

$168 \quad 63-81 \%$ of named partners were tested for syphilis as a result of disease intervention

169 specialist investigations. ${ }^{19-21}$ In randomised controlled trials of patients with gonorrhoea or

170 chlamydial infection, conducted in the United States, $35-52 \%$ of named partners either tested

171 negative or were treated thorough simple patient referral. ${ }^{22,23}$ Another previous randomised

172 controlled trial among men and women with chlamydial infection found that $45 \%$ of named

173 partners were treated by simple patient referral. ${ }^{24}$ Our study found that in Japan the proportion

174 of partners tested through simple patient referral was only $13 \%$ among married couples. This 
Second edition

175 result is similar to the result of the previous study conducted in Japan, but is lower than those

176 of studies conducted in other countries. One possible reason for this difference may be that

177 physicians in Japan may not explain the necessity of spousal testing to patients more carefully

178 compared with those in other countries. Because PN services involving public health sectors

179 have not been not implemented in Japan, there may be a difference in physicians' interest

180 and practice in caring for partners. As a result, some physicians may not adequately inform

181 patients regarding the need for partner testing. In addition, because the present study was

182 conducted among married couples, disclosing a diagnosis of syphilis to a partner would have

183 revealed infidelity; therefore, patients may have been reluctant to disclose the diagnosis to

184 their spouse. These factors may partially explain the differences in the proportion of partners 
Second edition

185 tested in our study and previous studies.

186 Patients may have difficulty in referring their partner for syphilis testing if the

187 physician advises, "Tell your partner that he/she needs to be tested." because patients are

188 hesitant to notify their partner due to guilt and stigma. ${ }^{25}$ Therefore, public health specialists

189 need to support patients' efforts to communicate with their partners by helping them

190 understand the need to notify their partners and the possible adverse health effects of having

191 an untreated STI. In a retrospective observational study of PN of syphilis conducted in

192 Switzerland, patients diagnosed at a public university hospital were less likely to notify their

193 partner than those diagnosed at other institutions. ${ }^{26}$ This difference may have been due to

194 differences in patient characteristics between institutions. In contrast, in our study, all 12 


\section{Second edition}

195 spouses of index patients treated at hospitals were tested for syphilis. Because the health care

196 setting in Japan allows patients to visit any medical institution, it may be due to differences

197 between hospitals and clinics in the quality of $\mathrm{PN}$, rather than differences in the

198 characteristics of the patient population.

200 cases, the present study showed that the proportion of wives aged 20-44 years who were

201 tested for syphilis declined during the study period. The Japanese Ministry of Health, Labour,

202 and Welfare has strengthened its prevention programs in order to increase public awareness.

203 This effort has included new types of health campaigns that use manga cartoons to target the

204 public in their 20 s to 40 s and increase opportunities for people to undergo STI testing. ${ }^{27}$ 
Second edition

205 However, health information campaigns and a population-based intervention approach are

206 only effective among those who consider themselves to be at risk. ${ }^{28}$ Therefore, the decrease

207 in spousal testing, despite health campaigns, may be due to an increased incidence of syphilis

208 in populations previously at low risk.

Our study has several limitations. Firstly, information about the syphilis stage of the

210 index patients was unavailable. Unlike the high infectivity of primary and secondary syphilis,

211 the transmission of late syphilis is unlikely; thus, screening contacts of patients with late

212 syphilis is generally unnecessary. However, even in patients with late-latent syphilis, it is

213 appropriate to screen long-term sex partners for syphilis. ${ }^{7-10}$ Because our study used marital

214 data, screening of spouses was advisable regardless of the syphilis stage of the index patient. 
Second edition

215 Secondly, the nature of the marital relationship during the infectious period was unclear. If

216 the patient did not have sexual contact with the spouse during the infectious period, the

217 outcomes of spousal syphilis testing might be underestimated as the spouse did not need to

218 be tested for syphilis. In particular, it was estimated that more older couples had not had

219 sexual contact in the preceding two years compared with younger couples. Thirdly, selection

220 bias may limit the generalisability of our findings because the population of employees and

221 their spouses in the present study had a higher socioeconomic status than the overall

222 population at risk of syphilis. However, given that patients with syphilis are less likely to

223 notify casual partners than long-term stable partners, ${ }^{29}$ the contacts of patients with syphilis

224 in Japan overall may be less likely to undergo screening than the population of our study. 
Second edition

225 Fourthly, the small sample size of index patients precludes a multivariate analysis of partner

226 testing according to variables such as sex, age, clinical characteristics, or provider type.

227 However, the data used in the present study were from the largest database of information on

228 married couples that is currently available in Japan. To address the public health concern of

229 the rapid increase in the incidence of syphilis, our study provides timely results to public

230 health policymakers from a current database. Finally, spouses may have undergone syphilis

231 testing that was not covered by health insurance. People can visit specialised medical

232 institutions at relatively low prices under the Japanese health insurance system. ${ }^{30,31}$ Although

233 some local public health centres are trying to improve access to free syphilis testing, the

234 accessibility to free testing is generally poor. In addition, most syphilis cases have been 


\section{Second edition}

235 reported from clinics and hospitals rather than from local public health centres.

236 Despite public health interventions to increase syphilis testing of contacts in Japan, ${ }^{3}$

237 only approximately one-seventh of spouses of patients who start syphilis treatment undergo

238 testing. The present study provides evidence that syphilis testing among contacts has been

239 low and has declined over the past eight years. Although surveillance and awareness-raising

240 have been intensified in Japan due to the recent surge in the incidence of syphilis, there is a

241 need to coordinate surveillance and field services that remain fragmented. Our findings

242 highlight the importance of introducing PN services that will result in higher partner testing

243 rates. 
Second edition

244 Acknowledgements: We thank Editage (www.editage.jp) for English-language editing.

245

246 Conflict of interest: The authors declare no conflicts of interest relevant to this article.

247

248 Funding: The authors disclosed receipt of the following financial support for the research,

249 authorship, and/or publication of this article: This work was supported by JSPS KAKENHI

250 Grant Number 19K19353 and Kyoto University Research Fund for Young Scientists (Start-

$251 \mathrm{Up}$ ).

252 
Second edition

253

254 1. Centers for Infectious Disease Control and Prevention. Sexually Transmitted Disease Surveillance 2017. Atlanta: Department of Health and Human Services, 2018.

2. Public Health England. Sexually transmitted infections (STIs): annual data tables,

257 https://www.gov.uk/government/statistics/sexually-transmitted-infections-stis-annualdata-tables (2010, accessed 26 July 2019).

3. Takahashi T, Arima Y, Yamagishi T, et al. Rapid Increase in Reports of Syphilis Associated With Men Who Have Sex With Women and Women Who Have Sex With

261 Men, Japan, 2012 to 2016. Sex Transm Dis 2018; 45: 139-143.

262 4. National Institute of Infectious Diseases. Infectious Disease Annual Report 2014, 


\section{Second edition}

263 https://www.niid.go.jp/niid/ja/allarticles/surveillance/2270-idwr/nenpou/6142-

264 syulist2014.html (2015, accessed 26 September 2019; In Japanese)

265 5. National Institute of Infectious Diseases. Summary of syphilis notifications in Japan,

266 https://www.niid.go.jp/niid/images/epi/syphilis/2018q4/syphilis2018q4.pdf $(2019$,

267 accessed 26 July 2019; In Japanese).

268 6. Ferreira A, Young T, Mathews C, et al. Strategies for partner notification for sexually

269 transmitted infections, including HIV. Cochrane Database Syst Rev 2013; 3: 1.

270 7. Workowski KA, Bolan GA. Centers for Disease Control and Prevention. Sexually

271 transmitted diseases treatment guidelines, 2015. MMWR Recomm Reports 2015; 64: 1272137. 
Second edition

273 8. Kingston M, French P, Higgins S, et al. UK national guidelines on the management of

274 syphilis 2015. Int J STD AIDS 2016; 27: 421-446.

275 9. Public Health Agency of Canada. Canadian Guidelines on Sexually Transmitted

276 Infections: Management and Treatment of Specific Infections - Syphilis,

277 https://www.canada.ca/en/public-health/services/infectious-diseases/sexual-health-

278 sexually-transmitted-infections/canadian-guidelines/sexually-transmitted-

279 infections/canadian-guidelines-sexually-transmitted-infections-27.html (2016, accessed

$280 \quad 26$ July 2019).

281 10. Australasian Sexual Health Alliance. Australian STI Management Guidelines for Use in

282 Primary Care. Syphilis, http://sti.guidelines.org.au/sexually-transmissible- 
Second edition

283 infections/syphilis (2018, accessed 26 July 2019).

284 11. Valentine JA, Bolan GA. Syphilis Elimination. Sex Transm Dis 2018; 45: S80-85.

285 12. Kimura S, Sato T, Ikeda S, et al. Development of a Database of Health Insurance Claims:

286 Standardization of Disease Classifications and Anonymous Record Linkage. J Epidemiol

$287 \quad 2010 ; 20: 413-419$.

288 13. Tanaka S, Seto K, Kawakami K. Pharmacoepidemiology in Japan: Medical Databases

289 and Research Achievements. J Pharm Heal Care Sci 2015; 1: 16.

290 14. Japanese Society for Sexually Transmitted Infections. STI Diagnosis and Treatment

291 Guidelines 2011. Japanese J Sex Transm Infect http://jssti.umin.jp/pdf/guideline-

292 2011.pdf (2011, accessed 26 July 2019; In Japanese). 
Second edition

293 15. Japanese Society for Sexually Transmitted Infections. STI Diagnosis and Treatment

294 Guidelines 2016. Japanese J Sex Transm Infect http://jssti.umin.jp/pdf/guideline-

295 2016.pdf (2016, accessed 26 July 2019; In Japanese).

296 16. World Health Organization. WHO Guidelines for the Treatment of Treponema pallidum

297 (Syphilis). Geneva: WHO, 2016.

298 17. Statistics Bureau, Ministry of Internal Affairs and Communications. Portal Site of

299 Official Statistics of Japan. https://www.e-stat.go.jp/ (2019, accessed 26 September 2019;

$300 \quad$ In Japanese).

301 18. Kodama T, Nakase K, Tsuda T, et al. Factors affecting appropriate management of 
Second edition

19. Heumann CL, Katz DA, Dombrowski JC, et al. Comparison of In-Person Versus outcomes by reported use of internet-based apps to meet sex partners in North Carolina,

310 21. Avoundjian T, Stewart J, Peyton D, et al. Integrating Human Immunodeficiency Virus

311 Testing Into Syphilis Partner Services in Mississippi to Improve Human 
Second edition

312 Immunodeficiency Virus Case Finding. Sex Transm Dis 2019; 46: 240-245.

313 22. Kissinger P, Mohammed H, Richardson-Alston G, et al. Patient-Delivered Partner

314 Treatment for Male Urethritis: A Randomized, Controlled Trial. Clin Infect Dis 2005; 41:

$315 \quad 623-629$.

316 23. Golden MR, Whittington WLH, Handsfield HH, et al. Effect of Expedited Treatment of

317 Sex Partners on Recurrent or Persistent Gonorrhea or Chlamydial Infection. $N$ Engl J

$318 \quad$ Med 2005; 352: 676-685.

319 24. Low N, McCarthy A, Roberts TE, et al. Partner Notification of Chlamydia Infection in

320 Primary Care: Randomised Controlled Trial and Analysis of Resource Use. BMJ 2006; 
Second edition

$321 \quad 332: 14-19$.

322 25. Gorbach PM, Aral SO, Celum C, et al. To notify or not to notify. Sex Transm Dis 2000;

$323 \quad 27: 193-200$.

324 26. de Lorenzi C, Gayet-Ageron A, Girard-Strohbach M, et al. Tracing partners of patients

325 with syphilis infection remains challenging: experience of Geneva Hospital. Int J STD

$326 \quad$ AIDS 2017; 28: 1090-1097.

327 27. Hiroshi Naruse, Kazuaki Jindai, Tomoya Saito. Fictional heroes take on real public health

328 problems: Japan's use of manga and anime in health campaigns,

329 https://blogs.bmj.com/bmj/2019/06/11/fictional-heroes-take-on-real-public-health-

330 problems-japans-use-of-manga-and-anime-in-health-campaigns/ (2019, accessed 26 July 
Second edition

$3312019)$.

332 28. Frohlich KL, Potvin L. Transcending the Known in Public Health Practice. Am J Public

333 Health 2008; 98: 216-221.

334 29. Gursahaney PR, Jeong K, Dixon BW, et al. Partner Notification of Sexually Transmitted

335 Diseases: Practices and Preferences. Sex Transm Dis 2011; 38: 821-827.

336 30. Ikegami N, Campbell JC. Japan's Health Care System: Containing Costs and Attempting

337 Reform. Health Aff 2004; 23: 26-36.

338 31. Hashimoto H, Ikegami N, Shibuya K, et al. Cost Containment and Quality of Care in

339 Japan: Is There a Trade-Off? Lancet 2011; 378: 1174-1182. 
Second edition

\section{$341 \quad$ Figure legends}

342

343 Figure 1. Flow diagram of the participant selection process

344 
Second edition

Table 1. Characteristics of the index patients $(n=217)$.

\begin{tabular}{lrl}
\hline Characteristics & n (\%) \\
\hline Age, years & & \\
$20-44$ & 89 & $(41.0)$ \\
$45-74$ & 128 & $(59.0)$ \\
Female sex & 35 & $(16.1)$ \\
STI history & & \\
Gonorrhoea & 6 & $(2.8)$ \\
Chlamydia & 12 & $(5.5)$ \\
Genital herpes & 19 & $(8.8)$ \\
History of psychiatric disorders & 12 & $(5.5)$ \\
Age of spouse, years & & \\
20-44 & 101 & $(46.5)$ \\
$45-74$ & 116 & $(53.5)$ \\
Provider type & & \\
Clinic & 205 & $(94.5)$ \\
Hospital & 12 & $(5.5)$ \\
Year of syphilis treatment & & \\
2010-2011 & 14 & $(6.5)$ \\
$2012-2013$ & 15 & $(6.9)$ \\
$2014-2015$ & 58 & $(26.7)$ \\
2016-2017 & 130 & $(59.9)$ \\
\hline
\end{tabular}

STI, sexually transmitted infection 
Second edition

Table 2. Number and proportion of index patients whose spouses underwent syphilis testing according to patient characteristics

\begin{tabular}{|c|c|c|c|c|c|c|}
\hline \multirow[b]{2}{*}{ Variables } & \multicolumn{3}{|c|}{ Male index patients } & \multicolumn{3}{|c|}{ Female index patients } \\
\hline & & $\begin{array}{l}n=182 \\
(\%)\end{array}$ & $P$ & & $\begin{array}{l}n=35 \\
(\%)\end{array}$ & $\boldsymbol{P}$ \\
\hline Overall & 23 & $(12.6)$ & & 6 & $(17.1)$ & \\
\hline \multicolumn{7}{|l|}{ Age, years } \\
\hline $20-44$ & 13 & $(19.4)$ & $0.062 *$ & 4 & $(18.2)$ & $>0.99 *$ \\
\hline $45-74$ & 10 & $(8.7)$ & & 2 & $(15.4)$ & \\
\hline \multicolumn{7}{|c|}{ History of STIs } \\
\hline No & & $(13.9)$ & $0.377^{*}$ & 4 & (12.9) & $0.128 *$ \\
\hline Yes & 2 & $(6.5)$ & & 2 & $(50.0)$ & \\
\hline \multicolumn{7}{|c|}{$\begin{array}{l}\text { History of psychiatric } \\
\text { disorders }\end{array}$} \\
\hline No & 21 & (12.2) & $0.617^{*}$ & 6 & $(18.2)$ & $>0.99 *$ \\
\hline Yes & 2 & $(20.0)$ & & 0 & $(0.0)$ & \\
\hline \multicolumn{7}{|c|}{ Age of spouse, years } \\
\hline $20-44$ & 14 & $(16.5)$ & $0.181^{*}$ & 3 & $(18.8)$ & $0.654 *$ \\
\hline $45-74$ & 9 & $(9.3)$ & & 3 & $(15.8)$ & \\
\hline \multicolumn{7}{|c|}{ Provider type } \\
\hline Clinic & 14 & $(8.1)$ & $<.0001 *$ & 3 & (9.4) & $0.003 *$ \\
\hline Hospital & 9 & $(100.0)$ & & 3 & $(100.0)$ & \\
\hline \multicolumn{7}{|c|}{ Years of syphilis treatment } \\
\hline 2010-2011 & 3 & $(37.5)$ & $0.014 \dagger$ & 2 & $(33.3)$ & $0.547 \dagger$ \\
\hline 2012-2013 & 3 & $(25.0)$ & & 0 & $(0.0)$ & \\
\hline 2014-2015 & 6 & $(13.0)$ & & 2 & $(16.7)$ & \\
\hline 2016-2017 & 11 & $(9.5)$ & & 2 & $(14.3)$ & \\
\hline
\end{tabular}

*Fisher's exact test

$\dagger$ Cochran-Armitage test

STI, sexually transmitted infection 
Second edition

Table 3. Syphilis testing among wives aged 20-44 years, according to the characteristics of the index patient

\begin{tabular}{lrrll}
\hline Variables & $\begin{array}{c}\text { Number of } \\
\text { spouses }\end{array}$ & $\begin{array}{c}\text { Syphilis } \\
\text { testing }\end{array}$ & $\mathbf{( \% )}$ & $\boldsymbol{P}$ \\
\hline Overall & 85 & 14 & $(16.5)$ & \\
Age of the index patient, years & & & & \\
$20-44$ & 65 & 13 & $(20.0)$ & $0.171^{*}$ \\
$45-74$ & 20 & 1 & $(5.0)$ & \\
History of STIs & & & & \\
No & 69 & 13 & $(18.8)$ & $0.290^{*}$ \\
Yes & 16 & 1 & $(6.3)$ & \\
History of psychiatric & & & & \\
disorders & & & & \\
No & 81 & 13 & $(18.8)$ & $0.520^{*}$ \\
Yes & 4 & 1 & $(25.0)$ & \\
Provider type & & & & \\
Clinic & 80 & 9 & $(11.3)$ & $<0.001^{*}$ \\
Hospital & 5 & 5 & $(100.0)$ & \\
Years of syphilis treatment & & & & \\
$2010-2011$ & 3 & 2 & $(66.7)$ & $0.026 \dagger$ \\
$2012-2013$ & 6 & 2 & $(33.3)$ & \\
$2014-2015$ & 21 & 3 & $(14.3)$ & \\
$2016-2017$ & 55 & 7 & $(12.7)$ & \\
\hline
\end{tabular}

*Fisher's exact test

$\dagger$ Cochran-Armitage test

STI, sexually transmitted infection 
4,024 patients with ICD-10 code A51-A53

between January 2010 and December 2017

1,142 patients without syphilis testing

1,643 patients without antibiotics for syphilis

$1,239(30.8 \%)$ patients with syphilis

24 patients under age 19

154 patients without health insurance plan six months before treatment

49 patients received surgery or blood transfusion

33 patients with endoscopy

3 patients with coronary angiography

$976(24.3 \%)$ eligible patients with syphilis

708 patients whose spouses do not have the same health insurance 5 patients whose spouses had been previously treated for syphilis

46 patients without spouse health insurance for three months after diagnosis

$217(5.4 \%)$ patient-spouse data included in analysis

Figure 1. Flow diagram of the participant selection process.

Figure 1. Flow diagram of the participant selection process.

$426 \times 299 \mathrm{~mm}(300 \times 300 \mathrm{DPI})$ 